\section{REVISTA}

Actualidades Investigativas en Educación http://revista.inie.ucr.ac.cr/

ISSN 1409-4703

\title{
BENEFICIOS DE LA MÚSICA EN CONDUCTAS DISRUPTIVAS EN LA ADOLESCENCIA
}

BENEFITS OF MUSIC IN DISRUPTIVE BEHAVIOURS DURING ADOLESCENCE

\section{Volumen 15, Número 3}

Setiembre - Diciembre

pp.1-24

\section{Este número se publicó el $1^{\circ}$ de setiembre de 2015}

DOI: http://dx.doi.org/10.15517/aie.v15i3.20902

Rocío Chao Fernández

María Dorinda Mato Vázquez

Vicente López Pena

Revista indizada en REDALYC, SCIELO

Revista distribuida en las bases de datos:

CATÁLOGO DE LATINDEX, IRESIE, CLASE, DIALNET, DOAJ, E-REVIST@S, SHERPA/ROMEO, QUALIS, MIAR

Revista registrada en los directorios:

ULRICH'S, REDIE, RINACE, OEI, MAESTROTECA, PREAL, CLACSO 


\title{
BENEFICIOS DE LA MÚSICA EN CONDUCTAS DISRUPTIVAS EN LA ADOLESCENCIA \\ BENEFITS OF MUSIC IN DISRUPTIVE BEHAVIOURS DURING ADOLESCENCE
}

\author{
Rocío Chao Fernández ${ }^{1}$ \\ María Dorinda Mato Vázquez \\ Vicente López Pena ${ }^{3}$
}

Resumen: El presente artículo analiza los beneficios de la música en la modificación de la conducta, la autoconfianza, la empatía, la autoestima y las habilidades sociales, fruto de la experiencia realizada durante un curso escolar con un grupo de discentes de 4o curso de Enseñanza Secundaria Obligatoria (12-16 años) en La Coruña (España). Ante la imposibilidad de desarrollar con éxito la labor docente debido al retraso académico de los alumnos y su indisciplina se decidió poner en práctica en el aula ordinaria un modelo de trabajo diseñado a partir de la recopilación de conocimientos de pedagogía musical, musicoterapia e inteligencia emocional. Para su desarrollo se han empleado diferentes instrumentos diagnósticos (basados en los tests formulados por Gallego, Alonso, Cruz y Lizama), con el fin de, una vez obtenidos los resultados, replantear la metodología de trabajo a través del empleo de música clásica, y comprobar las mejoras en los parámetros anteriormente señalados. Este nuevo planteamiento otorga un carácter innovador a la enseñanza musical, y en él apostamos por la importancia de descubrir estrategias motivadoras en el proceso de enseñanza-aprendizaje. Tras la puesta en práctica de esta experiencia confirmamos la hipótesis de que a través de la música es posible mejorar el comportamiento, ya que tras emplear las estrategias adecuadas, puede utilizarse como potenciadora de conductas tolerantes. A través de la aplicación de nuevos test y de los resultados académicos se corroboró que el 100\% de la muestra mejoró su autoestima, la autoconfianza, sus habilidades sociales, se redujeron los conflictos, y comenzaron a realizar trabajos en conjunto, así fueron modificando sus conductas disruptivas o antisociales.

Palabras clave: MÚSICA, ADOLESCENCIA, CONDUCTA, AUTOESTIMA, EMPATÍA, EDUCACIÓN SECUNDARIA, ESPAÑA

\begin{abstract}
This article aims to analyze the benefits of Music in the modification of behaviour, self-confidence, empathy, self-esteem and social abilities, as a result of an experience performed during a school year carried out with a secondary education class (4th year of Obligatory Secondary Education 12-16 years) in Coruña (Spain). Due to the impossibility to develop successfully the educational work, due to academic delay students and their discipline it was decided to put into practice in regular classroom a work model designed after putting together knowledge in the fields of music pedagogy, music therapy, and emotional intelligence. To develop this work, several instruments have been used to diagnose the problems and, once the results were obtained, to redesign the methodology through classical music and check the in the parameters mentioned before through Music. This new approach gives an innovative character to musical teaching, and with it we bank on the importance of finding new motivating strategies in the teaching-learning process. After putting this experience into practice, we can confirm the hypothesis that through music you can improve performance, as the results show the benefits that can come from the use of music as a tool in the modification of certain behaviours, since using the right strategies it may be used as an enhancer of tolerant behaviours, through the application of new test and academic performance as it has been corroborated that $100 \%$ of the sample improved their self-esteem, self-confidence, their social abilities, the number of conflicts decreased and the students started working in groups.
\end{abstract}

Key Words: MUSIC, ADOLESCENCE, BEHAVIOUR, SELF-ESTEEM, EMPATHY, HIGH SCHOOL, SPAIN.

\footnotetext{
${ }^{1}$ Profesora Contratada Doctora de Didáctica de la Expresión Musical en la Facultad de Ciencias de la Educación de la Universidad de A Coruña, España. Dirección electrónica: rocio.chao@udc.es

2 Profesora Contratada Doctora de Didáctica de la Matemática en la Facultad de Ciencias de la Educación de la Universidad de A Coruña, España.Dirección electrónica: $\underline{\text { m.matov@udc.es }}$

${ }^{3}$ Profesor en la Escuela Superior de Ingeniería de la Universidad de Cádiz, España.Dirección electrónica: vicente.lopez@uca.es
}

Artículo recibido: 8 de julio, 2014

Enviado a corrección: 30 de enero, 2015

Aprobado: 17 de agosto, 2015 


\section{Introducción}

La literatura científica y educativa lleva muchos años presentando importantes investigaciones de reconocidos y prestigiosos autores, como Dalcroze (1970), Hemsy de Gainza (2002a, 2002b), Swanwick (1997), Tomatis (1991), Vallés Arándiga (2003) y Willems $(1970,1979,1981)$ entre otros, quienes dedicaron y siguen ofreciendo buena parte de su trabajo a buscar respuestas científicas e innovadoras a diferentes momentos del desarrollo evolutivo del discente a lo largo de su formación.

Para ir situando el tema, diremos que el concepto de Inteligencia Emocional, aunque esté de actualidad, tiene un claro precursor en el concepto de Inteligencia Social del psicólogo Edward Thorndike (1920, p. 228) quien la definió como "la habilidad para comprender y dirigir a los hombres y mujeres, muchachos y muchachas, y actuar sabiamente en las relaciones humanas". Para Thorndike, además de la inteligencia social, existen también otros dos tipos de inteligencias: la abstracta -habilidad para manejar ideas- y la mecánica- habilidad para entender y manejar objetos-.

Un ilustre antecedente cercano de la Inteligencia Emocional lo constituye la teoría de 'las inteligencias múltiples' del Dr. Howard Gardner, de la Universidad de Harvard, quien plantea que las personas tenemos 7 tipos de inteligencia que nos relacionan con el mundo. Esta teoría introdujo dos tipos de inteligencias muy relacionadas con la competencia social, y hasta cierto punto emocional: la Inteligencia Interpersonal y la Inteligencia Intrapersonal.

Aunque el primer uso del término Inteligencia Emocional se atribuye a Payne (1986), pues lo menciona en su tesis doctoral, a saber, "Un estudio de las emociones: El desarrollo de la inteligencia emocional", fue Goleman (1996), investigador y periodista del New York Times, quien lo popularizó a través de su obra "La Inteligencia Emocional" (1996) problema se encuentra a la hora de valorar la Inteligencia Emocional (I.E.) de una persona, pues a diferencia de lo que ocurre con los test que miden el cociente intelectual $(\mathrm{Cl})$, según Goleman (1996, 1999, 2003), no existe un test capaz de determinar el grado de I.E. Él proporciona una detallada guía para fomentarla y robustecerla, pero no explica cómo podemos evaluar nuestro grado de I.E. concreto. Por su parte el Dr. Gallego ha llevado a cabo una amplia y rigurosa investigación, fruto de la cual nació el libro "Implicaciones educativas de la Inteligencia Emocional", (Gallego, Alonso, Cruz y Lizama, 1999), en el que incluye:

- Un test para diagnosticar el nivel en cada una de las 7 Inteligencias referidas por Gardner (1998). 
- Un test de diagnóstico de la Inteligencia Emocional global.

- Un test que analiza cada uno de los pilares de la Inteligencia Emocional (autoconciencia, autocontrol, automotivación, empatía o habilidades sociales) y cómo influyen nuestras emociones a la hora de tomar decisiones.

Por otra parte, los valores educativos y terapéuticos de la música son conocidos desde la antigüedad, pero es especialmente en el Siglo $X X$ cuando se comienzan a realizar investigaciones más rigurosas. Kodály (citado en Chao, 2010) desarrolló una de las primeras investigaciones rigurosas en las que demostró los ámbitos en los que influía positivamente el estudio de la música. Actualmente diversos autores (Campbell, 1998; Chao, Mato y Ferreiro, 2014; Fonseca y Toscano 2012; Levitin, 2011; Rusinek, 2004) consideran que la educación musical es un ámbito de intervención pedagógica que debe ser abordado desde la formación general del individuo, ya que entre las capacidades desarrolladas a través del estudio de la música, se pueden destacar las siguientes: potencia el razonamiento inductivo/deductivo, favorece el lenguaje y estudio de lenguas extranjeras, influencia en el pensamiento lógicomatemático, mejora la atención, la respiración, la relajación, la motricidad fina, el autoconcepto, la empatía, el sentido de responsabilidad, la perseverancia, el compromiso, entre otros (Campbell, 1998 y 2000; Despins, 1986; Goleman, 1996; Shapiro, 1997). En definitiva, según Pérez y Leganés (2012, p.129) "la experiencia musical activa la imaginación y la creatividad, construyendo el fundamento desde el cual actúan los procesos de cognición, percepción, atención, memoria, inteligencia, pensamiento y lenguaje".

Las primeras referencias al uso terapéutico de la Música en España se remontan al siglo XVIII, y han sido documentadas en la literatura por Poch (1999a, 1999b). En la década de los sesenta la Dra. Serafina Poch introdujo en España la Musicoterapia como profesión y fue la primera autora de una Tesis Doctoral sobre este tema en este país. En 1975 se organizó en Madrid el primer curso de Introducción a la Musicoterapia dirigido por el Dr. Benenzon, que dio pie a la formación de la Asociación Española de Musicoterapia (AEMT). Aunque se han realizado varias investigaciones en el ámbito de la musicoterapia financiadas por organismos públicos y privados, esta disciplina en España aún se reconoce por su carácter eminentemente práctico en el que la investigación y la teorización quedan, por el momento, en un segundo plano. 


\section{Objetivo}

El objetivo principal de esta investigación es analizar cuál es el punto de partida o perfil de un grupo de alumnos de $4^{\circ}$ de Educación Secundaria Obligatoria (sus carencias, déficits, dificultades, motivaciones) y tratar de averiguar si a través de una metodología diferente e innovadora, combinando los beneficios de la Música y el trabajo de la Inteligencia Emocional, es posible mejorar su comportamiento, reducir el desarrollo de conductas de riesgo y fortalecer el afrontamiento de situaciones difíciles.

Con base en el objetivo principal, se han establecido los siguientes objetivos secundarios: Ayudar al alumnado a mejorar la confianza en si mismo y la autoestima mediante la autorrealización; Mejorar la empatía del estudiantado; Mejorar las aptitudes, habilidades sociales e integración grupal del alumnado (tanto en relaciones interpersonales como intrapersonales); Modificar el paisaje sonoro del grupo; Reducir el nivel acústico de escucha; Establecer un gusto por la música diferente a la habitual en los componentes del grupo, y hasta ahora desconocida; Desinhibirse a la hora de cantar, bailar o tocar un instrumento.

\section{Metodología}

\subsection{Muestra}

La investigación se realizó a lo largo del curso 2013/14 con un grupo de 7 discentes de 4ํ de Educación Secundaria Obligatoria (en adelante, 4ำ de ESO) que estaban totalmente desmotivados y con conflictos entre ellos, se realizó una diagnosis exhaustiva de cada uno la cual comenzó por un informe realizado por el Departamento de Orientación y se concluyó con la aplicación de varios test para evaluar su Inteligencia Emocional.

1. A. B. G. 17 años (repite $4^{\circ}$ E.S.O.). En ocasiones acude al centro custodiado por la policía pues recibe agresiones físicas y psicológicas de su padre.

2. D.C.D. 17 años (repite $4^{\circ}$ E.S.O.). Intento de suicidio el curso pasado. Tiene 4 casos de suicidio similares en su familia.

3. B. C. S. 17 años (repite $4^{\circ}$ E.S.O.). Introvertida, con baja autoestima. Nunca estuvo bien integrada en ningún grupo. Problemas de autocontrol, con gran dependencia al tabaco y alguna sustancia prohibida.

4. J. L. S. 17 años (repitió $3^{\circ}$ E.S.O.). Baja autoestima, no está bien integrado en el grupo/clase. Interrumpe constantemente las clases. Desconocimiento absoluto de conductas sociales elementales. 
5. A. M .P. 18 años. (Repite 4ํㅡㄹ E.S.O, y anteriormente otro curso). Introvertido. Le cuesta integrarse en su grupo/clase. No interrumpe, pero tampoco participa. No tiene ansias de futuro. No le interesa el sistema educativo ordinario. Desmotivado.

6. S. P. M. 16 años. Equilibrada y extrovertida. Siempre estuvo bien integrada.

7. J. P. P. (Repite $4^{\circ}$ E.S.O, y anteriormente otro curso) Familia desestructurada con padre alcohólico y malos tratos físicos. Fue siempre un alumno problemático y disruptivo.

\subsection{Instrumentos}

Todos los instrumentos de diagnóstico utilizados fueron tomados del libro Implicaciones Educativas de la Inteligencia Emocional (Gallego et al. 1999).

\subsubsection{Test de las siete inteligencias. (Gallego et al. 1999, p.18)}

Este test se basa en las publicaciones del profesor Howard Gardner (1998) donde refleja los estudios realizados a adolescentes, y mediante los cuales llega a la conclusión de que existe un número desconocido de capacidades humanas. El objetivo con la aplicación de este test es diagnosticar en qué tipo de inteligencia destaca cada discente.

\subsubsection{Test de la Inteligencia Emocional global (Gallego et al. 1999, p. 45)}

El concepto de inteligencia emocional se debe a Meter Salovey y Jonh Mayer (en Gallego et al.1999) y está especificado tan solo desde principios de los años 90 . Con este nombre se incluyen 2 conceptos básicos: la inteligencia intrapersonal y la interpersonal, destacando dentro de ellas 5 habilidades, que también reconoce Goleman (1996, 1999, 2003). Estas son: autoconciencia, autocontrol, automotivación, empatía y habilidades sociales. Estas cualidades no son innatas a las personas, por lo que en cada caso se puede establecer el nivel de inteligencia emocional, y por supuesto pueden proponerse estrategias de mejora para conseguir la obtención de hábitos emocionales correctos.

3.2.3 Test de automotivación: “¿Tiene suficiente confianza en sí mismo?” (Gallego et al. 1999, p.125)

Según Goleman (1996), la automotivación es la capacidad de motivarse para perseguir unos objetivos o logros. 
3.2.4 Test de Empatía: ¿Se interesa por las personas? (Gallego et al. 1999, p. 52)

A juicio de Goleman (1996) la empatía es "la capacidad de reconocer las emociones ajenas, entender lo que otras personas sienten, así como comprender pensamientos y sentimientos que no se hayan expresados verbalmente" (en Gallego et. al; 1999, p.39).

3.2.5 Test de autoconciencia: ¿Muestra abiertamente sus emociones? (Gallego et al. 1999, p. 62)

La autoconciencia es "el conocimiento o reconocimiento de las propias emociones. Solo quien sabe por qué se siente como se siente, puede manejar sus emociones, moderarlas y ordenarlas de manera consciente". Es la base de la inteligencia emocional, ya que el hecho de conocer nuestro estado emocional servirá para controlar otras habilidades (Gallego et al. 1999, p. 39).

3.2.6 Test de autocontrol: “¿Cuál es el grado de dominio sobre sí mismo?”. (Gallego et al. 1999, p.77)

Según Goleman (1996), el autocontrol es "la capacidad de controlar las emociones y adecuarlas al momento y a las circunstancias. No podemos desconectarnos o evitar algunas emociones, pero sí podemos conducir nuestras respuestas emocionales y manejarlas de forma inteligente" (en Gallego et. al; 1999, p. 40). Poseer este tipo de habilidad implica dominar las propias emociones y sentimientos y en definitiva poseer ciertas habilidades que permiten a las personas, decidir correctamente, reaccionarse ante los acontecimientos,...se trata de buscar el equilibrio entre las emociones positivas y negativas, ya que todas ellas enriquecen nuestra inteligencia emocional.

3.2.7Test de habilidades sociales. (Gallego et al. 1999, p.180)

El concepto de habilidades sociales es difícil de determinar, pero en definitiva se podría decir que es la capacidad de conocer las reglas que rigen los comportamientos sociales. Según Gallego et al. (1999, p.183), consiste en

conocer cuándo y cómo hay que expresar los sentimientos propios y ajenos, cuándo hay que mostrar autoafirmación, cómo saber mantener una conversación, terminarla [...] Las habilidades sociales son comportamientos emocionales o conductas que se manifiestan en las relaciones interpersonales y que tienen la característica de ser socialmente bien aceptados. 


\subsection{Procedimiento}

Se intentó hacer un diagnóstico de la inteligencia emocional (habilidades sociales, empatía, autoestima....). Para ello, una vez estudiados los componentes del grupo de manera individual, se decidió aplicar los test señalados anteriormente.

El procedimiento llevado a cabo fue el siguiente:

- Se realizó un análisis de los test con el objeto de conocer los aspectos más acentuados del alumnado que pudiesen repercutir o causar las conductas disruptivas.

- Se diseñó una metodología pedagógico- musical para abordar los problemas detectados.

- Se pasaron de nuevo los test para comprobar si se modificaron las actitudes y la conducta.

Los primeros test se aplicaron en el mes de septiembre y los segundos a finales de mayo, después de que se les hubiese presentado una visión diferente del aprendizaje musical, a través de la vivencia activa de descubrimientos y reflexiones, realizando actividades y viviendo el aprendizaje como una experiencia progresiva, divertida y formativa.

\subsubsection{Análisis de los primeros test}

Con base en el objetivo principal de esta investigación se analizan los puntos de partida o perfil de este grupo de $4^{\circ}$ de ESO (sus carencias, déficits, dificultades, motivaciones, entre otros).

Test de las siete inteligencias.

A continuación se detallan los resultados obtenidos por el alumnado en lo referente a las siete inteligencias: Lingüística (L.), Lógica Matemática (L.M.), Espacial (E.), Musical (M.), Kinésico corporal (K.), Interpersonal (IE) e Intrapersonal (IA):

- Inteligencia lingüística. Según Gallego et al. (1999, p. 18):

Llamar a la capacidad lingüística "inteligencia" es coherente con la postura de la psicología tradicional. [...] El cerebro tiene un área específica, perfectamente conocida, llamada "área de Broca", responsable de la producción de oraciones gramaticales... Las personas bien dotadas de capacidad lingüística pueden explicar, debatir, persuadir 
con eficacia. Se pueden expresar de palabra o por escrito utilizando los medios de comunicación.

Para valorar los resultados, se atiende a lo expuesto al final del manual de referencia (Gallego et al, p.264) "si se obtiene 4 puntos o más indica una capacidad natural en ese tipo de inteligencia. Si puntúa 2 o menos en una categoría quiere decir que ese tipo de inteligencia está poco desarrollada".

En este parámetro sólo S.P.M. ha obtenido un 4 en este tipo de capacidad, por lo que es la única que tiene desarrollada este tipo de inteligencia.

- Inteligencia Lógico-matemática. Atendiendo a Gallego et al. (1999, pp. 18-19):

En los individuos dotados de este tipo de inteligencia el proceso de resolución de problemas es, a menudo, extraordinariamente rápido. El científico competente maneja simultáneamente muchas variables y crea numerosas hipótesis que son evaluadas sucesivamente y posteriormente aceptadas o rechazadas. Los estudiantes con un importante componente de inteligencia Lógico-matemática suelen tener éxito en los estudios.

En esta inteligencia ningún miembro del grupo llega a la puntuación de 4, necesaria para considerarla desarrollada; el máximo alcanzado es 2 puntos, por lo que creemos que la tienen poco trabajada.

- Inteligencia Espacial, a juicio de Gallego et al. (1999, p. 20): "Este tipo de inteligencia se relaciona con tareas como lectura de mapas, visualización de un objeto visto desde ángulos diferentes, antes visuales..."

En nuestros resultados, solo S.P.M. ha obtenido un 4, teniendo el resto del grupo esta inteligencia poco desarrollada.

- Inteligencia Musical. Según Gallego et al. (1999, p. 21) "La capacidad musical es una de las habilidades más tempranas, que emerge en el niño. La música es una buena herramienta de aprendizaje. [...] La fuerza de la inteligencia musical innata varía de persona a persona. Sin embargo es evidente que debe estimularse y orientarse para lograr su pleno desarrollo." 
Atendiendo a los resultados obtenidos, se desprende que ninguno tiene desarrollada este tipo de inteligencia.

- Inteligencia Kinésico-corporal. Teniendo en cuenta la opinión de Gallego et al. (1999, pp.2022) es:

La habilidad para utilizar el cuerpo de diferentes formas, para expresarse o para llevar a cabo una tarea. [...] La habilidad para competir en un juego (como ocurre en los deportes), para utilizar su propio cuerpo (como en la danza) o para crear un producto (como el diseño de un invención) constituye la evidencia de las características cognitivas del uso corporal.

La única persona que tiene desarrollada este tipo de inteligencia es A. M. P., que obtuvo una puntuación elevada (5), a mucha distancia del resto de la clase.

- Inteligencia interpersonal. Según Gallego et al. (1999, pp. 22-23)

La inteligencia interpersonal es la destreza para comprender a los otros, motivarles, cooperar con ellos. Se construye a partir de una capacidad para sentir distinciones entre los demás: en particular contrastes en sus estados de ánimo, temperamento, motivaciones e intenciones. Esta inteligencia capacita al adulto para leer las intenciones, emociones y deseos de los demás, aunque sean ocultos. En épocas anteriores se creía que este tipo de inteligencia era innato. Hoy pensamos que se trata, en gran parte de capacidades adquiridas, que se pueden enseñar, aprender y practicar.

Analizando los resultados del test, ninguno de los 7 discentes tiene desarrollada esta destreza, siendo la puntuación en todos los casos muy baja.

- Inteligencia intrapersonal. Teniendo en cuenta a Gallego et al. (1999, pp. 18-22)

Es un complemento natural de la inteligencia interpersonal. Es la capacidad de introspección, de formar una imagen adecuada de lo que uno es y de cómo se comporta. En línea con las teorías de Freud, se trata de autoentenderse, acceder y comprender los propios sentimientos y emociones, controlarlos y enfocarlos adecuadamente, orientando la propia conducta. Una persona con buena inteligencia 
interpersonal posee un modelo viable y eficaz de sí mismo. [...] La inteligencia intrapersonal permite comprenderse y trabajar con uno mismo...

En este parámetro sólo S.P.M. ha obtenido un 4 en este tipo de capacidad, por lo que es la única que tiene desarrollada este tipo de inteligencia.

\section{Test de la Inteligencia Emocional Global}

Una vez aplicado el test de la Inteligencia Emocional, se obtuvieron los siguientes resultados (Tabla 1).

Tabla 1. Test de la inteligencia emocional global

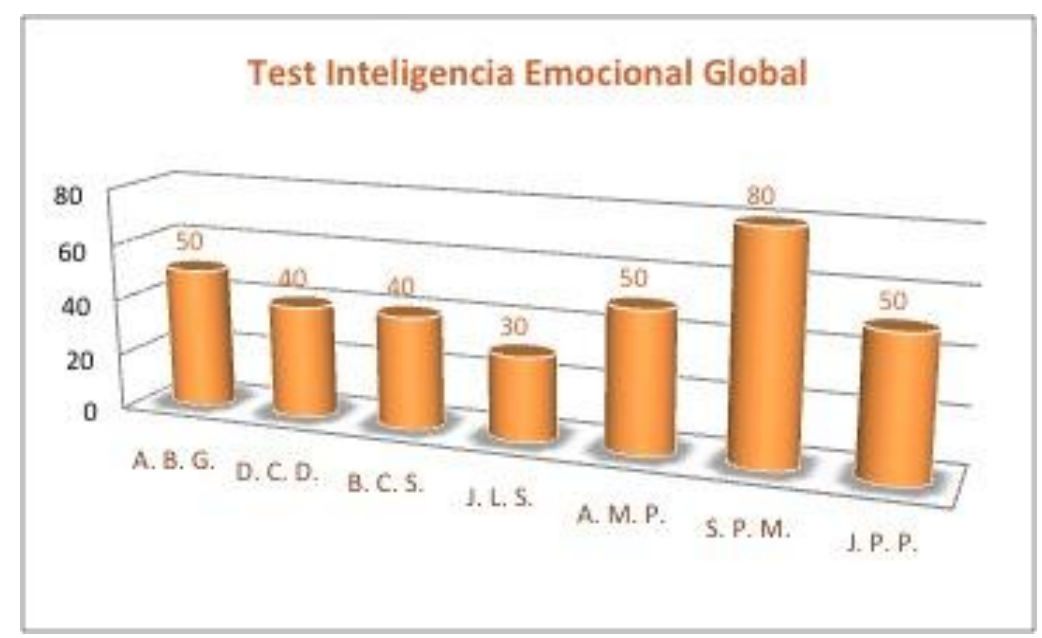

Fuente: elaboración propia

Para evaluar los resultados, según Gallego et al. (1999, p. 46) hay 3 categorías:

- Hasta 60 puntos "Su inteligencia emocional se encuentra por debajo de los límites normales". En esta situación se encuentran todos los miembros del grupo, excepto S.P.M.

- Hasta 80 puntos: "Su inteligencia emocional se encuentra dentro de los márgenes normales, pero siempre es aconsejable hacer una reflexión sobre nuestros puntos fuertes y débiles para superarse". En este lugar se encuentra S.P.M.

- Más de 120 puntos: "Dispone de un elevado grado de inteligencia emocional, pero tal vez la lectura de estas unidades le ayude a enseñar a otros a conseguirlo". En este parámetro no se encuentra ninguno de los 7 discentes. 
Test de automotivación: "¿Tiene suficiente confianza en sí mismo?”

En lo relativo a este test los resultados son los siguientes: A.B.G., 4; D.C.D., 3; B.C.S., 3; J.L.S, 4; A.M.P., 4; S.P.M., 9; J.P.P., 4.

Para evaluar los resultados, según Gallego et al. $(1999$, p. 126) hay 4 categorías:

- Menos de 5 puntos: Usted es una persona interesada y deseosa de aprender. Pero su autoconfianza no es en estos momentos muy grande. Se siente dependiente de otras personas y, peor aún, usted mismo entierra su propia seguridad. Probablemente es demasiado crítico (y demasiado autocrítico). Debería concentrarse más en los puntos fuertes y no en los débiles.

En este lugar se encuentra todo el grupo, excepto S.P.M

- Entre 5 y 9 puntos:

Su autoconfianza no es mayor ni menor que la de la mayoría de las personas, pero podría ser mucho mejor. Tal vez considere egoísta buscar la seguridad en sí mismo pero tenga en cuenta que las personas con una buena autoconciencia pueden dar ánimo y fuerza a los demás. Y esto no es en absoluto ser egoísta.

La puntuación de S.P.M se encuentra dentro de esta categoría.

- Entre 10 y 14 puntos (Gallego et al, 1999, p. 127) no hay ningún discípulo.

Su autoconfianza en tan fuerte que los demás no pueden hacerle nada malo. Pero examine de una manera más autocrítica sus puntos fuertes a nivel espiritual y compruebe si no ha erigido un sólido muro a su alrededor en el que ningún enemigo (pero tampoco algunos amigos) puede penetrar.

- Más de 14 puntos .En este grupo no hay ningún discente. (Gallego et al, 1999, p. 127) En la mayoría de situaciones de la vida se muestra seguro y equilibrado. Esto no quiere decir que tenga menos problemas que las otras personas, sólo que reacciona de manera diferente ante ellos. Los asume, intenta lo mejor y reconoce que una persona no siempre puede tener éxito ni permanecer en el centro de atención. En esto se diferencia de las personas que aparentan autoconfianza pero que en realidad se sienten tremendamente inseguros.

Test de Empatía: ¿Se interesa por las personas?

Se obtuvieron los siguientes resultados (Tabla 2): 
Tabla 2. Test para averiguar el nivel de empatía

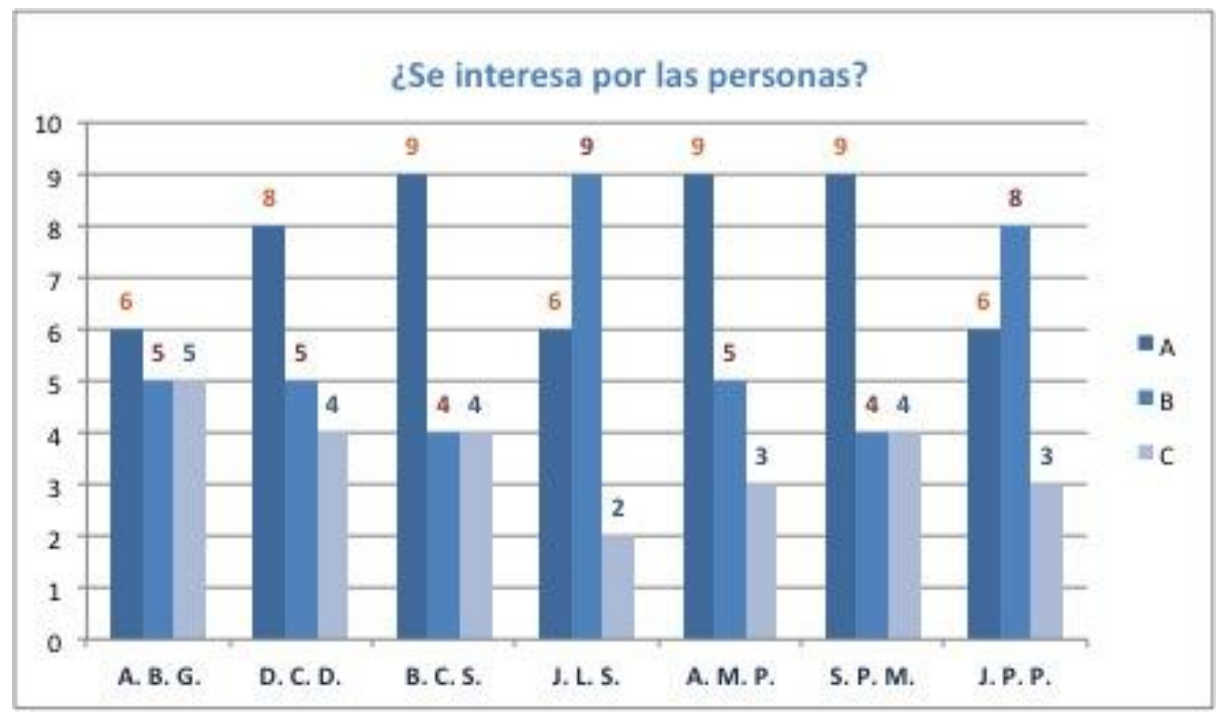

Fuente: elaboración propia

A. B. G.; D. C.D.; B. C.S.; A. M.P. y S. P.M. tienen una mayoría de respuestas A, por lo que a juicio de Gallego et al. (1999, p. 53)

Es de esas personas con las que siempre se puede contar. Su preocupación por cuestiones ajenas hace que se tenga mucha confianza en ellos. Incluso se puede llegar a fijar más en lo del otro que en sus propios problemas. Siempre está para lo que hace falta, escucha cuando se lo piden y es un buen consejero. Sin duda, es un buen amigo/a. Pero cuidado. Tanta preocupación e interés por cuestiones ajenas se puede volver en su contra ya que a todo el mundo no le tiene por qué gustar su forma de pensar y puede desembocar en problemas. Ayuda a quien quiera ser ayudado.

J. L. S. y J. P.P. obtuvieron una mayor puntuación en las respuestas $B$, por lo que según Gallego, Alonso, Cruz y Lizama (1999, p. 53), son personas que les motiva preocuparse de cuestiones relacionadas con su propia persona. Pero tampoco rechaza ayudar a otro que lo necesita aunque esa persona debe ser de confianza porque su colaboración no la ofrece a todo el mundo. Incluso, algunas veces colabora y, posteriormente y de forma inconsciente, es posible que lo saque a relucir delante de otras personas para que éstas lo conozcan. Esto es un grave error. Si da, no espere recibir, será mucho más feliz.

Ninguno obtuvo mayoría de respuestas tipo C. 
Test de autoconciencia: ¿Muestra abiertamente sus emociones?

Los resultados fueron los siguientes: A.B.G., 7; D.C.D., 5; B.C.S., 5; J.L.S, 6; A.M.P., 7;

S.P.M., 11; J.P.P., 6.

Todo el grupo, salvo S.P.M han obtenido menos de 8 puntos, lo que según Gallego et al. (1999, p. 63) indica que

usted es una persona sensiblemente emocional, pero también con oscilaciones en su estado de ánimo. Por eso los demás no saben cómo se les acercará y qué deben esperar de usted. Si es éste el caso, usted es caprichoso aunque no le guste la palabra. Caprichoso es el que se deja llevar por sus estados de ánimo y al que nadie puede valorar emocionalmente. A veces también le sucede que sólo quiere tener buenos sentimientos. Cuando también pueda aceptar en sí mismo pensamientos y sentimientos negativos, será cuando consiga un equilibrio tanto por fuera como por dentro.

La puntuación de S.P.M está en el rango de entre 8 y 12 puntos, lo que muestra que se esfuerza en mantener sus sentimientos bajo control. No se permite tener arrebatos ni con otras personas ni consigo mismo. Le gustaría mostrar siempre al mundo una cara sonriente. Pero así se está exigiendo demasiado. Todos tienen derecho a estar de mal humor y tan sólo deben manifestarlo para que los otros sepan lo que pasa. Si usted también lo hace, no se preocupará tanto por las propias emociones ya que usted y los demás sabrán que los arrebatos pueden tener lugar en cualquier momento.

Test de autocontrol: “¿Cuál es el grado de dominio sobre sí mismo?”: los resultados son los siguientes:

D.C.D.; B.C.S; J.L.S. han obtenido una puntuación inferior a 5 puntos, lo que significa que

es una persona demasiado impulsiva y no se controla cuando la gente dice o hace algo que le haga sentir mal. Debería controlar un poco más sus impulsos puesto que esas respuestas viscerales nunca son buenas para solucionar los problemas. Párese a pensar siempre antes de dar una respuesta definitiva.

J. P.P. obtuvo 6 puntos, por lo que según Gallego et al. (1999, pp. 78-79) 
tiene en cuenta todos los puntos a tratar en algunas situaciones, pero en otros momentos sus emociones se le escapan y no piensa lo que hace o dice delante de los demás. Debería estudiar sus puntos débiles y aprender a dominar esos impulsos.

Á. B.G. y S. P.M. obtuvieron 11 y 12 puntos respectivamente, por lo que aunque algunas veces sus emociones le tomen la delantera, lo normal es que las controle y analice reflexivamente. Cuando sus reacciones son impulsivas, sabe que se están produciendo y normalmente las enmienda. Sus reacciones ante los demás son sinceras y le sirven para una buena convivencia.

A.M.P. obtuvo 16 puntos, lo que significa que

su forma de ser tranquila, reconciliadora y pacífica le ayuda a superar cualquier obstáculo y es por ello por lo que sabe dominar sus emociones tanto si las cosas van bien como si van mal. Esta actitud causará admiración y confianza en los demás.

Test de habilidades sociales.

El análisis de este test demuestra que A.B.G. y D.C.D. poseen una personalidad inhibida (10 y 9 p.), B.C.S, J.L.S. y J.P.P inadecuada, A.M.P. inhibida y S.P.M. hábil.

En definitiva, el estudio de los test corrobora que en general el grupo carece de las habilidades sociales (quizás fundamentalmente por su baja autoestima) básicas para establecer relaciones grupales, que no saben comportarse en clase, ya que sus conductas son disruptivas (especialmente J. L. y J. P.) o excesivamente inhibidas (Á. B. o A. M.) y que carecen de empatía, por lo que no son capaces de reconocer los estados de ánimo o necesidades de sus compañeras y compañeros.

\subsection{Desarrollo del método empleado}

Dado que se estimó que sería imposible llevar a cabo con este alumnado la secuenciación habitual de la materia por varios motivos (por su comportamiento antisocial, porque tenían un interés nulo por el aprendizaje y porque partían de un nivel de desconocimiento total de la asignatura), se decidió reestructurar la metodología de trabajo teniendo en cuenta las peculiaridades del grupo, tomando como referencia las 
investigaciones realizadas por varios autores (Giné, 1997; Güell y Muñoz, 2003, etc.) sobre el pensamiento del alumnado de la ESO.

Una de las teorías que ha estudiado ampliamente el tema de la motivación escolar es la conocida como "La Teoría de la Motivación por Metas de Aprendizaje" (Alonso, 1998), que manifiesta que el alumnado aborda las demandas de la actividad escolar en función de la meta de aprendizaje que cada uno tenga trazada de antemano.

Además Bray (2000) realizó un estudio sobre la perspectiva que tiene el alumnado sobre el tipo de música que se trabaja en el aula, llegando a dos importantes conclusiones: la primera es que la materia no cubre las necesidades ni los intereses de los discentes de manera suficiente; la segunda es que la asignatura de música se percibe como una materia sin importancia para la vida académica.

Teniendo en cuenta las investigaciones anteriores, el segundo paso fue distribuir el espacio del aula en diferentes rincones: uno para las audiciones, otro para las improvisaciones instrumentales, otro para la expresión corporal, entre otros, para iniciar el trabajo a través de las audiciones, e introducirlos en un tipo de música diferente al habitual en ellos. Para llevarlo a cabo se utilizó una selección de obras de Wolfgang Amadeus Mozart, puesto que existen estudios (Tomatis, 1991 y Campbell, 1998) que demuestran que tranquiliza a sus oyentes, mejora la percepción espacial y les permite expresarse con mayor claridad, logrando con este tipo de música los mejores resultados y las reacciones más duraderas.

El trabajo de las primeras sesiones se inició a través de audiciones, utilizando obras de Mozart y dejando unos minutos al final de la clase para que un discente presentase al grupo el tipo de Música que le gusta. Para llevarlo a cabo tenían que negociar entre el orden de exposición. Pronto surgió otro inconveniente: estaban acostumbrados a escuchar música a un volumen demasiado elevado, lo que permitió reflexionar sobre el problema que esto supone y desconocían: en la actualidad hay estudios que avalan el hecho de que 60 millones de norteamericanos sufren pérdida de audición, y que un tercio de esas pérdidas están causadas por exposición a sonidos fuertes, como el simple hecho de escuchar la música tan fuerte como ellos lo hacen. El doctor Rosen (en Chao, 2005), médico del hospital Mount Sinai y profesor en la Universidad de Columbia de New York, ha hecho estudios comparativos en los que demuestra que en la sociedad africana tradicional cualquier persona de 60 años oye tan bien o mejor que un veinteañero norteamericano. A raíz de esto se 
planteó un nuevo objetivo: reducir paulatinamente el volumen de escucha, partiendo del nivel 26 al que estaban acostumbrados.

Progresivamente, además de audiciones de Mozart también se utilizó música de Haydn, que al igual que Mozart tiene claridad, elegancia y transparencia y ayuda a mejorar la concentración, la memoria y la percepción espacial, así como también la música barroca (Bach, Häendel, Vivaldi, Corelli), que induce a la estabilidad, orden y seguridad y genera un ambiente mentalmente estimulante para el estudio o el trabajo. Incluso en contadas ocasiones, bien avanzado el curso, se incluyeron cantos gregorianos, que según Tomatis (1991) crea sensación de espacio amplio y relajado; es excelente para el estudio y la meditación silenciosa; por lo que puede reducir el estrés.

Transcurridas las primeras cuatro sesiones era necesario incluir otro parámetro para trabajar, pero sin abandonar estos espacios para la audición, por lo que se estipuló que a lo largo del curso los primeros 10 minutos se dedicarían a escuchar música clásica y los 10 últimos, si la clase resultaba productiva, a escuchar la suya.

A partir de la quinta sesión comenzaron a realizar ejercicios de expresión corporal siguiendo el método de Dalcroze (1970), con los que se consiguió un objetivo fundamental: atraer su atención y que tuviesen ganas de seguir trabajando.

Tras varias sesiones en las que la profesora tocaba al piano y primero ellos caminaban libremente, a continuación debían moverse al ritmo que escuchaban (seguir el tempo natural -negras-, luego correr -corcheas-, entre otros), se les presentó la representación gráfica de los mismos ritmos a través cartones, para que conociesen las figuras y su valor.

En paralelo se realizaron ejercicios auditivos (con campanas, cascabeles y cencerros) extraídos del método Edgar Willems (1981) para trabajar con alturas cada vez más próximas, y ejercicios para ejercitar la memoria y la audición interior. También se incluyeron ejercicios para diferenciar la altura de los sonidos acompañados de los signos fononímicos de Zoltan Kodaly (en Chao, 2010). Asimismo se realizaron ejercicios de ritmos con percusión en las diferentes partes del cuerpo: palmas, dedos, nudillos, golpes en las rodillas, con los pies, entre otros. primero por imitación, y posteriormente con lecturas rítmicas sencillas. Estos ejercicios fueron evolucionando hasta llegar a hacer imitaciones espontáneas y naturales, utilizando varias sílabas onomatopéyicas, como: toc-toc-toc, tic, tic-tac,...etc.

La iniciación a la instrumentación se realizó a partir de un nuevo elemento: los boomwhackers, tubos sonoros de diferente longitud, y afinados según la escala de Do Mayor. Esta nueva incorporación resultó un éxito total, y un nuevo logro en nuestro 
propósito, ya que cada vez la seducción por la clase de música era mayor, además de mejorar la atención y la unión. Tanto es así que si en algún momento J. P. o J. L. (que eran los que tenían una conducta más primaria) se despistaban, eran sus propios compañeros quienes los convencían para continuar, y para que siguieran trabajando, ante el temor de que se modificase el desarrollo de las clases. Pronto interpretaron sencillas canciones ("Sal á ventana María”, "Campanita del lugar”, entre otras). Cuando aprendían a tocar las canciones con los boomwhackers, continuaron con los instrumentos de lámina, experiencia que también les apasionó.

En Navidades se organizaba un festival desde el Departamento de música, pero este grupo se negaba a participar. Sin embargo, la confianza en ellos mismos cada vez era mayor y estaban más satisfechos de comprobar que eran capaces de interpretar canciones. Un día la orientadora del centro entró en la clase de música mientras ellos tocaban y quedó tan impresionada del progreso, que les animó y finalmente decidieron participar.

El día de la actuación tocaron y cantaron varias canciones acompañados por los boomwhackers, y una versión muy simplificada y corta de la Sinfonía del Nuevo Mundo, adaptada para tocar con instrumentos de láminas en la escuela. Esto supuso un gran refuerzo de su autoestima, de la autoconfianza y cohesión del grupo, a la vez que les motivó para continuar trabajando en esta línea.

Además se propusieron preparar otra actuación para los Carnavales, lo que fue fundamental para afianzar el compañerismo, el trabajo en equipo, a la vez que su comportamiento mejoraba e todas las clases.

En paralelo, en el tiempo dedicado a escuchar su música, ya se redujo considerablemente el volumen de la escucha, que en estos momentos se situaba entre el nivel 16-17. A partir de los Carnavales, además de seguir con la línea habitual de trabajo, se realizaron las audiciones siguiendo el método de Jos Wuytack (1970), haciendo especial hincapié en el trabajo del ritmo, que implica organización y control. Según Lacarcel Moreno (1995) el ritmo es orden, ya que dispone los sonidos en el tiempo, sin él no existe periodicidad. Trabajado de manera adecuada, se puede conseguir que el orden que proporciona el ritmo pase a formar parte de la experiencia del alumnado, mejorando aspectos de su propia personalidad y percepción. 


\section{Resultados}

Tras llevar a cabo la metodología diseñada, se aplicaron de nuevo los test para comprobar qué mejoras se obtuvieron en los parámetros anteriormente señalados.

Test de las siete inteligencias (Tabla 3).

Tabla 3. Test de las siete Inteligencias

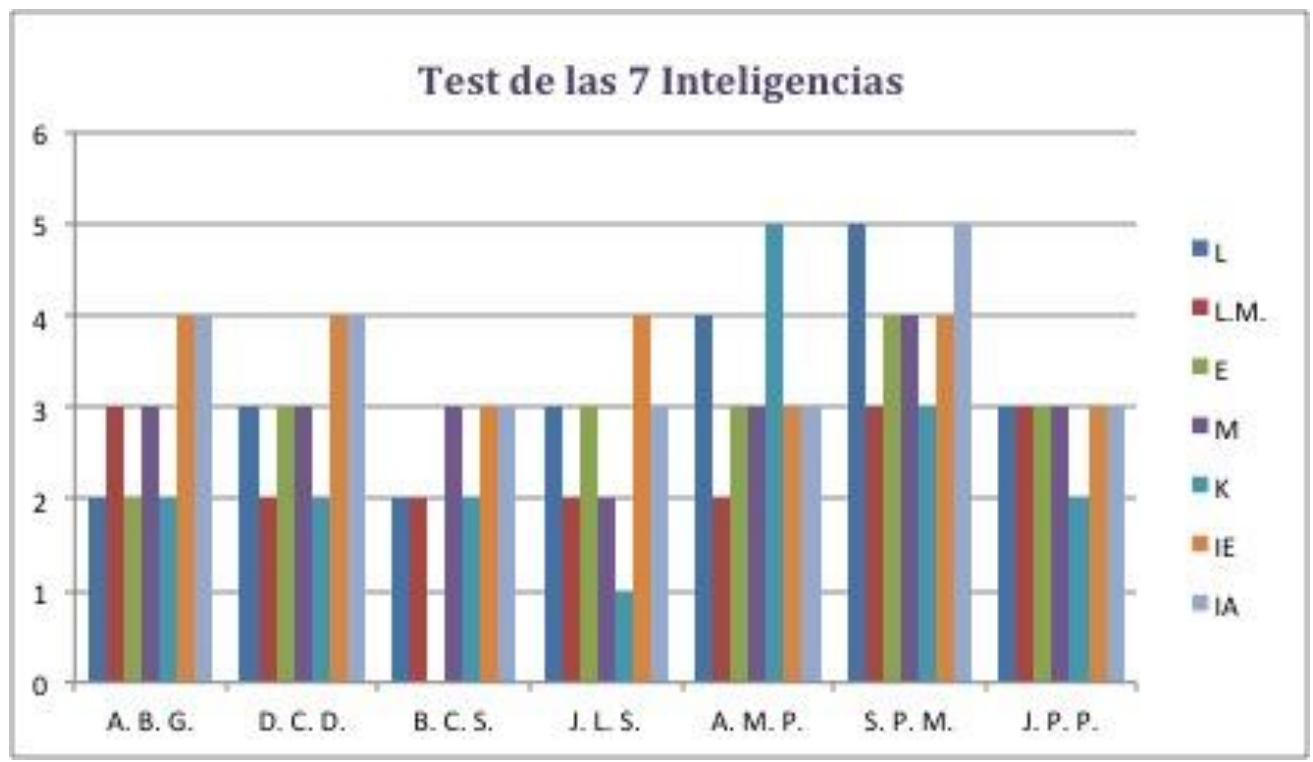

(Fuente: elaboración propia)

- -Inteligencia lingüística. Todo el grupo tiene una mejora considerable, aunque solo S.P.M. y A.M.P. obtienen una puntuación superior a 4.

- -Inteligencia Lógico-matemática. En este tipo de inteligencia todos mejoran su puntuación, aunque ninguno llega a tener desarrollado este tipo de inteligencia.

- Inteligencia Espacial. No hay modificaciones significativas.

- Inteligencia Musical. Ha mejorado la totalidad del grupo, aunque solo llega a 4 puntos S.P.M.

- Inteligencia Kinésico-corporal. No hay modificaciones significativas.

- Inteligencia interpersonal. Aquí es donde los resultado son más evidentes: todos han mejorado, y llegan a 4 puntos. A. B. G.; D. C. D.; J. L. S. y S.P.M.

- Inteligencia intrapersonal. Han mejorado especialmente A. B. G.; D. C. D. y S.P.M. 


\section{Test de la Inteligencia Emocional Global}

Todo el grupo ha mejorado su inteligencia emocional global, A.B.G. (70); A.M.P.(60); S.P.M. (90); J.P.P. (60), aunque 3 discentes continúan por debajo de los límites normales: D.C.D. (50); B.C.S. (50) y J.LS. (40).

Test de automotivación: “¿Tiene suficiente confianza en sí mismo?” En este tipo de inteligencia no se objetivaron modificaciones significativas.

Test de Empatía: ¿Se interesa por las personas? En este tipo de inteligencia no se objetivaron modificaciones significativas.

Test de autoconciencia: ¿Muestra abiertamente sus emociones? En este tipo de inteligencia no se obtuvieron modificaciones.

Test de autocontrol: “¿Cuál es el grado de dominio sobre sí mismo?"

Salvo A.M.P y S.P.M, (16 y 12), todos los demás han mejorado su puntuación A.B.G. (12);

D.C.D. (5); B.C.S. (5); J.P.P. (7) y solo J.L.S no llega a 5 puntos.

Test de habilidades sociales. En este tipo de inteligencia no se objetivaron modificaciones significativas, puesto que todo el alumnado confirma el tipo de personalidad que se perfiló en la primera valoración, aunque en el caso de J.L.S. y J.P.P se suaviza su perfil inadecuado, y en A.M.P. el inhibido. (Tabla 4). 
Tabla 4.- Test de habilidades sociales.

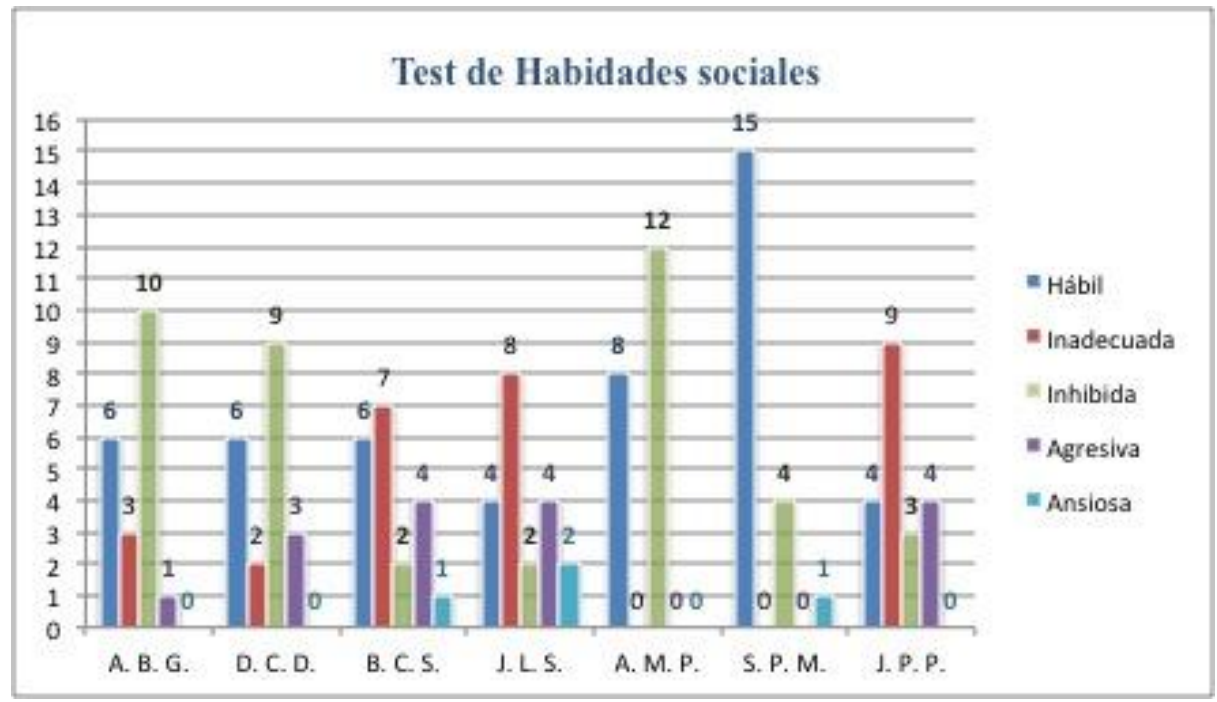

Fuente: elaboración propia

\section{Conclusiones}

La música es un fenómeno muy valorado por los adolescentes. Como tal, la oportunidad de escuchar o participar en actividades musicales se puede utilizar como refuerzo o premio en programas de modificación de conducta.

Los problemas emocionales del alumnado son una de las primeras causas de fracaso escolar, y aquí es donde el papel de la música se vuelve importante. A través de su práctica conseguimos que los discentes se relajen, y se olviden de sus problemas. Puede motivar, crear espíritu de grupo, ayudar a reforzar su personalidad, aumentar la autoestima, etc. La música enseña a ser constante, a trabajar para conseguir un objetivo. Es frecuente que los discentes de secundaria dejen muchas veces las tareas inacabadas, y a través de la práctica musical se proporcionan métodos para que aprendan a finalizar lo que hacen. $Y$ si se trata de tocar en grupo, es fundamental la implicación de todos para garantizar el éxito del proyecto. Aplicar este método en los estudios o las tareas de clase puede ser muy beneficioso.

Es en los momentos en que un discente problemático decide probar a esforzarse más para conseguir un aprobado cuando todos los implicados en su educación debemos estar alerta para ayudarle en lo posible. Debe comprender que, efectivamente, el esfuerzo ha valido la pena y que ha conseguido su objetivo. 
Trabajar con adolescentes problemáticos emocionalmente no ha sido un tema muy tratado en la literatura musical, y menos inscrito en el marco de la Educación Secundaria. Hemos tenido que extrapolar conceptos de la Musicoterapia en la Educación Especial, porque no existe mucha bibliografía explícita sobre la efectividad del trabajo con Música en conductas disruptivas en Educación Secundaria o sobre Musicoterapia y adolescencia. Tampoco hay estudios sobre la resolución de problemas emocionales en alumnado de Educación Secundaria sin discapacidades evidentes.

La presente investigación coincide con el estudio realizado por Ambrona, López y Márquez, 2012) que sugiere la necesidad de potenciar estas competencias y trabajar la inteligencia emocional desde la infancia, lo que otorga un carácter innovador, en la que apostamos por la importancia de descubrir estrategias motivadoras en el proceso de enseñanza-aprendizaje (Isasi, 2008).

En definitiva, teniendo en cuenta las premisas planteadas al inicio de la investigación: averiguar si, a través de la Música, se podía mejorar el comportamiento de los adolescentes con conductas disruptivas y antisociales, los resultados obtenidos fueron los siguientes:

- El grupo que recibió la intervención mejoró significativamente en todas las variables de medida.

- Se logró un aumento de la autoestima de los 7 discentes.

- Se logró una mejor integración de todo el grupo, lo que incidió en una reducción de los conflictos.

- Se logró aumentar su motivación en todos los ámbitos.

- Se consiguió que el grupo realizase trabajos en equipo.

- Se logró que mostrasen actitudes tolerantes.

- Se consiguió que participasen en actividades ante público.

- Se consiguió modificar su paisaje sonoro y reducir el nivel de escucha.

De todo ello consideramos que el trabajo más arduo fue conseguir que realizasen trabajos en equipo, basados en el respeto mutuo y la cooperación, imprescindibles en música para tocar una obra en conjunto. Es necesario señalar que antes del festival de Navidad se consiguió que si uno de ellos no trabajaba, o se equivocaba, eran sus propios compañeros los que le recriminaran, ya que llegaron a comprender que los fallos de uno repercutían en la globalidad del trabajo de los demás. 
Otro aspecto difícil fue convencer al grupo de que estaban preparados para dar a conocer a los demás los aprendizajes que estaban adquiriendo, y participasen en los festivales, ya que era un grupo infravalorado, tanto por el resto del alumnado, como por los profesores, pero una vez superado este hándicap, su participación en el festival fue muy positiva para ellos, pues adquirieron prestigio ante sus compañeros y ante el resto del profesorado. Este cambio de actitud no solo repercutió en el área de Música, sino que también se percibieron mejoras en otras áreas: aprendieron a respetar turnos de diálogo, a debatir para llegar a conclusiones o a discutir por conseguir un objetivo común, (como por ejemplo ponerse de acuerdo para escuchar un tipo de música), y a hacer críticas constructivas. Por tanto, si analizamos los resultados a partir de los objetivos planteados, se puede apreciar que la experiencia ha resultado satisfactoria.

En definitiva, se puede llegar a la conclusión de que la música puede mejorar determinadas conductas (Benezon, 2000; Bruscia, 1997 y 1999), ya que empleando las estrategias acertadas, puede emplearse como potenciadora de conductas tolerantes (como la solidaridad, la cooperación, el respeto mutuo), mejora de habilidades sociales, como el autoconcepto, la autoestima y empatía (Pena, Extremera y Rey, 2011), pero para conseguirlo, es necesario el compromiso y colaboración del profesorado (Betés de Toro, 2000).

A pesar de que los resultados de este estudio avalan la eficacia de esta metodología, somos conscientes de lo reducido de la muestra, por lo que consideramos que los resultados deberían ser contrastados en futuros estudios.

\section{Referencias}

Alonso Tapia, Jesús. (1998). Motivación y aprendizaje en el aula. Cómo enseñar a pensar. Madrid: Santillana.

Ambrona, Tamara, López-Pérez, Belén, Márquez-González, María. (2012). Eficacia de un programa de educación emocional breve para incrementar la competencia emocional de niños en educación primaria. Revista Española de Orientación y Psicopedagogía, 23(1), 39-49.

Benezon, Rolando. (2000). Musicoterapia. De la teoría a la práctica. Barcelona: Paidós.

Betés De Toro, Mariano (coord.). (2000). Fundamentos de musicoterapia. Madrid: Morata S.L.

Bray, Donald. (2000). An examination of GCSE music uptake rates. British journal of Music Education 17(1), 79-89. 
Bruscia, Kenneth. (1997). Definiendo Musicoterapia. Salamanca: Amarú.

Bruscia, Kenneth. (1999). Modelos de Improvisación en Musicoterapia. Vitoria: Agruparte.

Campbell, Donaldson. (1998). El Efecto Mozart. Barcelona: Ediciones Urano S.A

Campbell, Donaldson. (2000). El Efecto Mozart para niños. Barcelona: Ediciones Urano S.A.

Chao Fernández, Rocío. (2005). Fundamentos didácticos de los métodos pedagógicos del s. $X X$ a la actualidad. Villalba: Conservatorio Profesional Municipal de Música.

Chao Fernández, Rocío. (2010). La enseñanza del folklore gallego en los colegios de educación primaria de Galicia. Villalba: Conservatorio Profesional Municipal de Música.

Chao Fernández, Rocío, Mato Vázquez, Maㅡ Dorinda y Ferreiro, Francisco Jesús. (2014). Music Therapy in Adolescent Disruptive Behaviour. Procedia-Social and Behavioral Sciencies, 132, 608-614.

Dalcroze, Émile-Jacques. (1970). Le rythme, la musique et l'education. Milano: Hopli.

Despins, Jean Paul. (1986). La música y el cerebro. Barcelona: Gedisa.

Fonseca Mora, Carmen y Toscano Fuentes, Carmen María. (2012). La música como herramienta facilitadora del aprendizaje del inglés como lengua extranjera. Teoría de la Educación. Revista Interuniversitaria, 24(2), 197-213.

Gadner, Howard. (1998). Inteligencias Múltiples. Barcelona: Paidós.

Gallego, Domingo José, Alonso, Catalina María, Cruz, Ana y Lizama, Luis. (1999). Implicaciones Educativas de la Inteligencia Emocional. Madrid: UNED.

Giné, Núria, Maruny Curto, Lluís, Muñoz Martínez, Emili. (1997). Una canya o un pal? El pensament de l'alumnat sobre l'ESO. Barcelona: ICE de la UAB.

Goleman, Daniel. (1996). Inteligencia Emocional. Barcelona: Kairós.

Goleman, Daniel. (1999). La práctica de la Inteligencia Emocional. Barcelona: Kairós.

Goleman, Daniel. (2003). Emociones destructivas. Cómo entenderlas y superarlas. Barcelona: Kairós.

Güell Barceló, Manel y Muñoz Redon, Josep. (Coords.) (2003). Educación Emocional. Programa de actividades para Educación Secundaria Postobligatoria. Barcelona: Cisspraxis.

Hemsy de Gaínza, Violeta. (2002a). Música: Amor y conflicto. Buenos Aires: Lumen.

Hemsy de Gaínza, Violeta. (2002b). Pedagogía musical: dos décadas de pensamiento y acción educativa. Buenos Aires: Lumen. 
Isasi, Josefa. (2008). Desde el conocimiento ¿será posible motivar en el estudio? Madrid: Eride Ediciones.

Lacarcel Moreno, Josefa. (1995). Musicoterapia en Educación Especial. Murcia: Universidad de Murcia.

Levitin, Daniel. (2011). Tu cerebro y la música. Barcelona: RBA.

Payne, Wayne. (1986). A study of emotion: developing emotional intelligence; self integration; relating to fear, pain and desire. Dissertation Abstracts International, 47, 203A. (University microfilms No. AAC 8605928).

Pena Garrido, Mario, Extremera Pacheco, Natalio y Rey Peña, Lourdes. (2011). El papel de la inteligencia emocional percibida en la resolución de problemas sociales en estudiantes adolescentes. Revista Española de Orientación y Psicopedagogía, 22(1), 69-79.

Pérez Aldeguer, Santiago y Leganés Lavall, Esther Noemí. (2012). La Música como herramienta interdisciplinar: un análisis cuantitativo en el aula de Lengua extranjera de Primaria. Revista de Investigación en Educación, 10(1), 127-153.

Poch Blasco, Serafina. (1999a). Compendio de Musicoterapia (Volumen I). Barcelona: Empresa Editorial Herder, S.A.

Poch Blasco, Serafina. (1999b). Compendio de Musicoterapia (Volumen II). Barcelona: Empresa Editorial Herder, S.A.

Rusinek, Gabriel. (2004). Aprendizaje musical significativo. Revista Electrónica Complutense de Investigación en Educación Musical, 1(5), 1-16.

Shapiro, Lucy. (1997). La inteligencia emocional de los niños. Bilbao: Zeta.

Swanwick, Keith. (1997). Música, pensamiento y educación. Madrid: Morata.

Thorndike, Edward. (1920). Intelligence and its use. Harper's Monthly Magazine, 227-235.

Tomatis, Alfred. (1991). Pourquoi Mozart? Bélgica: Fixot.

Vallés Arándiga, Antonio. (2003). Psicopedagogía de la inteligencia emocional. Valencia: Promolibro.

Willens, Edgar. (1970). Introduction à la Musicotherapie. Bienne, Suiza: Eds.Pro Música.

Willens, Edgar. (1979). Las bases psicológicas de la educación musical. Buenos Aires: Eudea.

Willens, Edgar. (1981). El valor humano de la educación musical. Barcelona: Paidos.

Wuytack, Jos. (1970). Música Viva. Paris: A. Leduc. 